\title{
Polydisperse chains placed on a one-dimensional lattice
}

\author{
Jürgen F. Stilck * Minos A. Neto \\ Instituto de Física \\ Universidade Federal Fluminense \\ Av. Litorânea $s / n$ \\ 24210-340 - Niterói, RJ \\ Brazil
}

(Dated: November 9, 2018)

\begin{abstract}
Using a transfer matrix technique, we calculate the entropy of polydisperse chains placed on an one-dimensional lattice, as a function of the density of internal and endpoint monomers. The polydispersivity is determined considering different activities for the two types of monomers, as is usual for equilibrium polymerization and living polymers. If the mean number of monomers in the chains is fixed, the entropy as a function of the density of monomers displays a maximum and is limited from below by the entropy of monodisperse chains. The increase of entropy due to the polydispersivity is a linear function of the density of monomers. We also calculate the distribution of chain sizes, obtaining an exponential relation.

PACS numbers: 65.50.+m,05.20.-y
\end{abstract}

\section{INTRODUCTION}

In the microcanonical ensemble, the entropy of a system may be calculated by evaluating the number of microscopic configurations as a function of the internal energy and other extensive variables, such as the volume and the number of particles. The entropy, expressed in terms of these variables, is a fundamental equation of the system and thus all its thermodynamic properties may be obtained from this equation. A particular problem of this kind is the study of the thermodynamic properties of flexible chains placed on regular lattices. This problem has a rather long history, which reaches back to the thirties, where the thermodynamics behavior of diatomic molecules (dimers) adsorbed on surfaces was considered by Fowler and Rushbrooke [1]. In the simplest version of the model, only excluded volume interactions are taken into account, and thus each site of the lattice may be either empty or occupied by a single monomer. In this case, the internal energy of the system will be constant and the temperature is not defined. The dimer problem may be generalized by considering chains with more than two monomers. More precisely, we may define the entropy of a system of chains with $M$ monomers each ( $M$-mers) placed on a lattice with $V$ sites as

$$
s_{M}(\rho)=\frac{1}{V} \lim _{V \rightarrow \infty} \ln \Gamma\left(N_{p}, M ; V\right),
$$

where $\Gamma\left(N_{p}, M, V\right)$ is the number of ways to place $N_{p}$ chains with $M$ monomers in each on the lattice and the thermodynamic limit is taken with fixed density of occupied sites $\rho=N_{p} M / V$. In the particular case of dimers

\footnotetext{
*Electronic address: jstilck@if.uff.br

${ }^{\dagger}$ Electronic address: minos@if.uff.br

${ }^{\ddagger}$ Electronic address: wgd@if.uff.br
}

$(M=2)$ and fully covered $(\rho=1)$ two dimensional lattices, the entropy was calculated exactly [2], in other cases estimates were found through series expansions [3], closed form approximations [4] and transfer matrix calculations with finite size extrapolations [5].

Here we will study the one-dimensional version of the problem. After discussing briefly the monodisperse case, where all chains are composed by precisely $M$ monomers, we include the possibility of polydispersivity, allowing for a distribution of different numbers of monomers in chains. In particular, we consider an ensemble of polydisperse chains determined by different activities for endpointand internal monomers of the chains, such as is usual in models of equilibrium polymerization and living polymers [6, 7]. Perhaps the most studied experimental realization of equilibrium polymerization is the phase transition in liquid sulphur, and a good review of this and other experimental systems is due to Greer [8].

In the following section we will define the problem in more detail and show how it may be solved in the microcanonical ensemble. Section III presents the calculation in the grand-canonical ensemble, using a transfer matrix. Besides obtaining the entropy, we calculate also the distribution of chain sizes. Final discussions may be found in the conclusion.

\section{DEFINITION AND MICROCANONICAL SOLUTION OF THE MODEL}

Let us start with the formulation and solution of the monodisperse case. In this case we must calculate the number of ways to place $N_{p}$ chains with $M$ monomers each on a one-dimensional lattice with $L$ sites. Since in this case there are $L-M N_{p}$ empty sites, it is easy to conclude that this number is equal to

$$
\Gamma\left(N_{p}, M ; L\right)=\frac{\left(N_{p}+L-M N_{p}\right) !}{N_{p} !\left(L-M N_{p}\right) !} .
$$


Taking the thermodynamic limit $(L \rightarrow \infty)$ with the fraction of occupied sites $\rho=M N_{p} / L$ kept fixed and using Stirling's asymptotic form, we get

$$
\begin{aligned}
s_{M}(\rho)= & \left(\frac{\rho}{M}+1-\rho\right) \ln \left(\frac{\rho}{M}+1-\rho\right) \\
& -\frac{\rho}{M} \ln \frac{\rho}{M}-(1-\rho) \ln (1-\rho) .
\end{aligned}
$$

In Fig. 1] some curves of the entropy as a function of the density $\rho$ are shown. The entropy vanishes both at $\rho=0$ and $\rho=1$ and has a maximum at a density $\rho_{m}$ which grows monotonically with the molecular weight $M$, as may be also seen in the figure. In the polymer limit $M \rightarrow$ $\infty$ we find $\rho_{m}=1$, but the entropy vanishes identically.
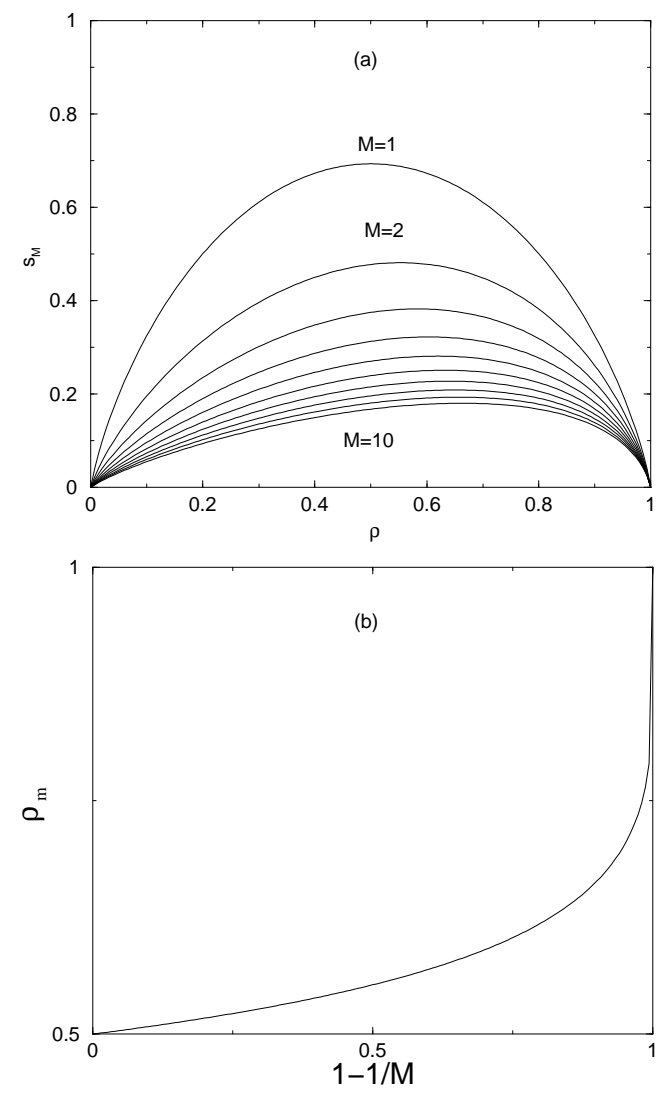

FIG. 1: a)Entropy as a function of the fraction of occupied sites for monodisperse chains with molecular weight $M$ between 1 and 10. b)Density of maximum entropy as a function of $1-1 / M$.

The entropy per site as a function of the density is a fundamental equation of the system, and all thermodynamic properties of this gas of $M$-mers may be obtained from it. Being athermal, the temperature is not defined for this gas, but the intensive entropic variables $(f / T$ and $\mu / T)$ may be obtained taking partial derivatives of $s_{M}(\rho)$. As an illustration, we will obtain the mechanical equation of state explicitly. Considering the lattice constant to be equal to $a$, the physical entropy will be $S=k_{B} a L s_{M}(\rho)$, where $k_{B}$ is the Boltzmann constant.
Since the system is one-dimensional, the pressure will be replaced by the force $f$ applied to the chain, therefore

$$
\frac{f}{T}=\frac{\partial S}{a \partial L}=\frac{k_{B}}{a}\left(s_{M}(\rho)-\rho \frac{d s_{M}}{d \rho}\right) .
$$

The result is

$$
\frac{f a}{k_{B} T}=\ln \left[1+\frac{\rho}{M(1-\rho)}\right] .
$$

It is interesting to expand the right side of this equation in powers of $\rho$, and the first terms are

$$
\begin{aligned}
\frac{f a}{k_{B} T}= & \frac{\rho}{M}+\frac{2 M-1}{2}\left(\frac{\rho}{M}\right)^{2} \\
& +\frac{1-3 M+3 M^{2}}{3}\left(\frac{\rho}{M}\right)^{3}+\cdots
\end{aligned}
$$

The first term corresponds to the ideal gas result, being equal to the number of $M$-mers divided by $L$. The higher order terms are all positive. In Fig. 2 the equations of state for dimers and tetramers are plotted. The linear behavior at low densities and the divergence as $\rho \rightarrow 1$ are apparent.

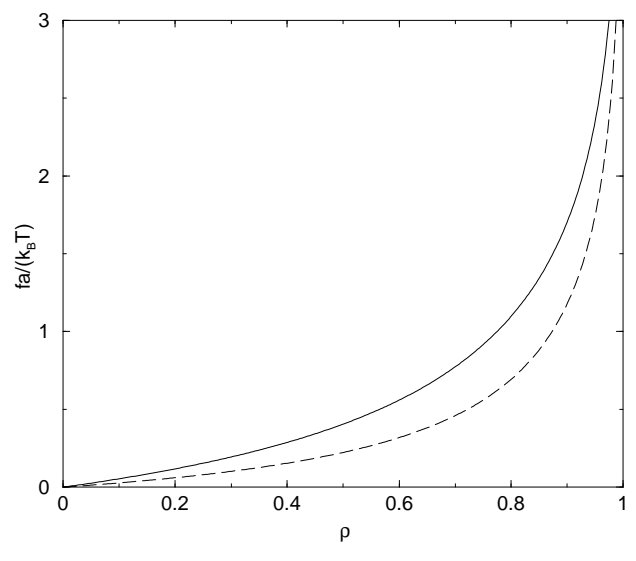

FIG. 2: Mechanical equation of state for dimers (full line) and tetramers (dashed line).

In equilibrium polymerization or living polymers, the chains grow through a process in two steps: first a chain is started and then it grows incorporating an additional monomer each time. In the case of liquid sulphur, the first step corresponds to the opening of a small ring if atoms (mainly $S_{8}$ ), followed by the opening and addition of other rings to the linear chain. This process may be parametrized by two activities or chemical equilibrium constants. The first one, $z^{\prime}=\exp \left(\mu^{\prime} / k_{B} T\right)$, is associated to the initial process and will be considered the statistical weight of chain endpoints. The second activity, $z=\exp \left(\mu / k_{B} T\right)$, relates to the addition of a monomer to an existing chain, and will be the statistical weight of internal monomers of the chains. $\mu$ and $\mu^{\prime}$ are the chemical potentials of internal and endpoint monomers, respectively [6]. Usually the propagating reaction which 
implies growing of the chains is faster than the opposite depropagating reaction, and this allows for the possibility of synthesizing chains with a very narrow distribution of molecular weights by artificially terminating the polymerization process, through the addition of an appropriate monomer [8]. If the equilibrium situation is reached, however, a polydisperse set of chains will be attained, and this is described by the simple model we consider with two activities.

In the particular case of sulphur, the endpoint monomer activity $z^{\prime}$ has a very small value, of the order of $10^{-12}$, and therefore very long chains are formed in this polymerization process. The equilibrium polymerization model may be mapped onto the $n$-vector model of magnetism, and the activity $z^{\prime}$ will be proportional to the magnetic field of the $n$-vector model in the limit $n \rightarrow 0$. Thus, in this case the polymerization is a continuous phase transition [6]. For finite values of $z^{\prime}$, where finite chains are dominant, no phase transition occurs, as we expect for a lattice gas of chains with excluded volume interactions only.

Now let us return to the calculation of the entropy in the one-dimensional model of equilibrium polymerization. The densities of endpoint monomers $x$ and internal monomers $y$ are conjugated to the activities $z^{\prime}$ and $z$, respectively. All configurations with the same numbers of chains $N_{p}=L x / 2$ and internal monomers $N_{i}=L y$ have the same statistical weight. We may then calculate the number of these configurations in a combinatorial way. Besides the multiplicity in Eq. (2), an additional factor corresponding to the number of ways to place the internal monomers in the $N_{p}$ chains is now present. The result is

$$
\begin{aligned}
\Gamma\left(N_{p}, N_{i} ; L\right)= & \frac{\left(L-N_{p}-N_{i}\right) !}{\left(L-2 N_{p}-N_{i}\right) ! N_{p} !} \times \\
& \frac{\left(N_{p}+N_{i}-1\right) !}{\left(N_{p}-1\right) ! N_{i} !} .
\end{aligned}
$$

In the thermodynamic limit, we find the following expression for the entropy per site

$$
\begin{aligned}
s(x, y)= & (1-x / 2-y) \ln (1-x / 2-y)- \\
& (1-x-y) \ln (1-x-y)-x \ln (x / 2)- \\
& y \ln y+(x / 2+y) \ln (x / 2+y) .
\end{aligned}
$$

The mean number of monomers per chain will be $\bar{M}=$ $2+2 y / x$, and if this number is fixed, we may express the entropy in the polydisperse case as a function of the density of monomers $\rho=x+y$. The result is

$$
\begin{aligned}
s_{\bar{M}}(\rho)= & \frac{\bar{M}(1-\rho)+\rho}{\bar{M}} \ln \left[\frac{\bar{M}(1-\rho)+\rho}{\bar{M}}\right]- \\
& (1-\rho) \ln (1-\rho)-\frac{2 \rho}{\bar{M}} \ln \left[\frac{2 \rho}{\bar{M}}\right]-\frac{\rho(\bar{M}-2)}{\bar{M}} \times \\
& \ln \left[\frac{\rho(\bar{M}-2)}{\bar{M}}\right]+\frac{\rho(\bar{M}-1)}{\bar{M}} \ln \left[\frac{\rho(\bar{M}-1)}{\bar{M}}\right](9)
\end{aligned}
$$

As expected, the entropy for the polydisperse case is never smaller than the one for the monodisperse case when $\bar{M}=M$. The difference between these entropies is

$$
\begin{aligned}
s_{\bar{M}}(\rho)-s_{M}(\rho)= & \frac{\rho}{M}[(M-1) \ln (M-1) \\
& -(M-2) \ln (M-2)] .
\end{aligned}
$$

The origin of the additional entropy in the polydisperse case is the second factor in expression 7.

Another point in this problem which may be addressed is the distribution of sizes of the chains. Again it is possible to answer this in a combinatorial way, if we consider the probability that a particular chain has $k=M-2$ internal monomers. This probability corresponds to the ratio between the number of internal monomer configurations when one of the $N_{p}$ chains has exactly $k$ internal monomers and the total number of configurations, which corresponds to the second factor in Eq. (7). In the thermodynamic limit, this leads to the result

$$
r_{k}=\frac{x}{x+2 y}\left[\frac{2 y}{x+2 y}\right]^{k},
$$

with $k=0,1,2, \ldots$. This result may be rewritten as

$$
r_{M}=\frac{1}{\bar{M}-1}\left[\frac{\bar{M}-2}{\bar{M}-1}\right]^{M-2}
$$

where $M=2,3,4, \ldots$ and $r_{M}$ is the fraction of chains with $M$ monomers, which is a function of the mean number of monomers in chains $\bar{M}$. It is worth to notice that the Flory-Huggins approximation to a lattice model for equilibrium polymerization also results in an exponential distribution of the chain sizes [7]. Both results are in fact equivalent, if one takes into account that Dudovicz et al. considered isolated sites to be chains with one monomer, while here we called them empty sites.

\section{TRANSFER MATRIX SOLUTION IN THE GRAND-CANONICAL ENSEMBLE}

The grand-canonical solution of the monodisperse case may be found in reference [5], so the polydisperse case will be discussed here. The equilibrium polymerization model in one dimension was discussed already in reference 9], with emphasis on the limit of infinite chains, where a phase transition occurs. Here we will use a similar transfer matrix technique to obtain the entropy and the distribution of chain sizes. To define a transfer matrix, we may use lattice gas variables $\eta_{i}=0,1$ associated to the bonds of the lattice, such that $\eta_{i}=1$ if the bond $i$ is inside a chain and $\eta_{i}=0$ otherwise. This definition is illustrated in Fig. 3] where a section of the lattice is depicted.

The transfer matrix for this case is given by

$$
T=\left(\begin{array}{cc}
1 & z^{\prime} \\
z^{\prime} & z
\end{array}\right)
$$




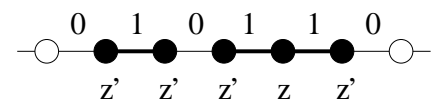

FIG. 3: Section of the lattice with a particular configuration of chains. The values of the bond variable $\eta$ are indicated above the line and below the line the activity of each monomer is given.

The largest eigenvalue of this matrix is

$$
\lambda_{1}=\frac{1+z+\sqrt{(1-z)^{2}+4\left(z^{\prime}\right)^{2}}}{2} .
$$

This eigenvalue may become degenerated when $z^{\prime}$ vanishes, originating the polymerization transition studied in reference 9]. For our present interest, we will consider finite values of $z^{\prime}$, so that no degeneracy occurs. In the thermodynamic limit, the grand-canonical potential will be given by

$$
\Phi=-k_{B} T V \ln \lambda_{1}
$$

and since the entropy $S$ is the partial derivative of $\Phi$ with respect to the temperature, and remembering that $z=\exp \left(\mu / k_{B} T\right)$ and $z^{\prime}=\exp \left(\mu^{\prime} / k_{B} T\right)$, we obtain the following expression for the adimensional entropy per site

$$
s=\frac{S}{k_{B} V}=\ln \lambda_{1}-\frac{z}{\lambda_{1}} \frac{\partial \lambda_{1}}{\partial z}-\frac{z^{\prime}}{\lambda_{1}} \frac{\partial \lambda_{1}}{\partial z^{\prime}} .
$$

Now we may recognize that

$$
y=\frac{z}{\lambda_{1}} \frac{\partial \lambda_{1}}{\partial z}
$$

is the density of internal monomers and

$$
x=\frac{z^{\prime}}{\lambda_{1}} \frac{\partial \lambda_{1}}{\partial z^{\prime}}
$$

is the density of endpoint monomers in chains. Some algebra will lead us to expression 8 for the entropy in the polydisperse case. In the limit $y \rightarrow 0$ we have $x=\rho$ and the entropy of dimers $s_{M=2}(\rho)$ is recovered.

To obtain the distribution of internal monomers among the chains, we may define an activities for the internal monomers which are dependent on the localization of the monomer in the chain. Thus, the leftmost internal monomer will have an activity $z_{1}$, his neighbor to the right has an activity $z_{2}$ and so on. The bonds of the lattice will also be numbered accordingly. A bond which is not inside a chain is represented by $\eta=0$, as before, but the $i$ 'th internal bond, counted from the left to the right, is associated to $\eta=i$. These definitions are illustrated in Fig. 4

The transfer matrix for the model now has an unlimited size, but a very simple structure

$$
T=\left(\begin{array}{ccccc}
1 & z^{\prime} & 0 & 0 & \cdots \\
z^{\prime} & 0 & z_{1} & 0 & \cdots \\
z^{\prime} & 0 & 0 & z_{2} & \cdots \\
\vdots & \vdots & \vdots & \vdots & \vdots
\end{array}\right)
$$

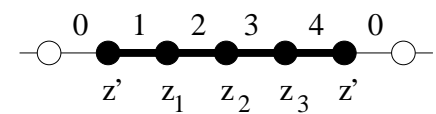

FIG. 4: Section of the lattice with a particular configuration of chains. The bond variables $\eta$ and monomer activities are shown, defined in a way to allow for the calculation of the size distribution of chains.

The secular equation may be found developing the determinant $|T-\lambda I|$ recursively by the first line. It is more convenient to express the equation in terms of $\omega=1 / \lambda$, and after some algebra, we get

$$
-1+\omega+\left(z^{\prime} \omega\right)^{2}\left(1+\sum_{i=1}^{\infty} \prod_{j=1}^{i} z_{j} \omega^{i}\right)=0 .
$$

Now the density of monomers in the $k^{\prime}$ th position in chains will be

$$
\rho_{k}=\frac{z_{k}}{\lambda} \frac{\partial \lambda}{\partial z_{k}}=-\frac{z_{k}}{\omega} \frac{\partial \omega}{\partial z_{k}} .
$$

Deriving the secular equation with respect to $z_{k}$ and then making all $z_{k}$ equal to $z$, we get

$$
\rho_{k}=\frac{\left(z^{\prime}\right)^{2} \omega(1-\omega z)}{(1-\omega z)^{2}+\left(z^{\prime}\right)^{2} \omega(2-\omega z)}(\omega z)^{k} .
$$

This result may be written as a function of the monomer densities $x$ and $y$

$$
\rho_{k}=\frac{x}{2}\left(\frac{2 y}{x+2 y}\right)^{k} .
$$

From this expression, the fraction $r_{k}$ of chains with $k$ internal monomers expressed in Eq. (11) may easily be found.

\section{CONCLUSION}

Although it was rather simple to find the entropy and distribution of chain sizes in the equilibrium polymerization model using combinatorial arguments in the microcanonical ensemble, it is interesting to perform these calculations also in the grand-canonical ensemble, since the combinatorial calculations are difficult to generalize to more complex situations, such as the problem defined on strips of finite widths (ladders). For the monodisperse case, such calculations have lead to rather precise estimates in the two-dimensional limit [5]. We are presently performing similar calculations for directed and self-avoiding chains on strips, which hopefully will allow us to estimate the properties of these models in the twodimensional limit. 


\section{Acknowledgments}

This work was supported by the brazilian agencies CNPq and FAPERJ. JFS acknowledges funding by project Pronex-CNPq-FAPERJ/171.168-2003.
[1] R. H. Fowler e G. S. Rushbrooke, Trans. Faraday Soc. 33, 1272 (1937).

[2] P. W. Kasteleyn, Physica 27, 1209 (1961); H. N. V. Temperley e M. E. Fisher, Philos. Mag. 6, 1061 (1961); M. E. Fisher, Phys. Rev. 124, 1664 (1961).

[3] A. M. Nemirovsky and M. D. Coutinho-Filho, Phys. Rev. A 39, 3120 (1989).

[4] J. F. Stilck and M. J. de Oliveira, Phys. Rev. A 42, 5955 (1990).

[5] W. G. Dantas and J. F. Stilck, Phys. Rev. E 67, 031803
(2003).

[6] J. C. Wheeler, S. J. Kennedy and P. Pfeuty, Phys. Rev. Lett. 45, 1748 (1980); J. C. Wheeler and P. Pfeuty, Phys. Rev. A 24, 1050 (1981).

[7] J. Dudowicz, K. F. Freed and J. F. Douglas, J. Chem. Phys. 111, 7116 (1999).

[8] S. C. Greer,J. Phys. Chem B 102, 5413 (1998).

[9] P. M. Pfeuty and J. C. Wheeler, Phys. Rev. A 27, 2178 (1983). 